\title{
P005. Efficacy Type-A Botulinum toxin treatment in a multidisciplinary setting for chronic headache
}

\author{
Giuseppe Vescio ${ }^{1}$, Amerigo Costa $^{2}$, Aida Squillace ${ }^{1}$, Rosario lannacchero ${ }^{2^{*}}$ \\ From Abstracts from the 1st Joint ANIRCEF-SISC Congress \\ Rome, Italy. 29-31 October 2015
}

\section{Background}

Type-A botulinum toxin (BoNTA) therapy has emerged as an effective treatment for chronic headache $(\mathrm{CH})$ [1]. No conclusive data exists about BoNTA efficacy in $\mathrm{CH}$ comorbid with anxiety and depression disorders. Our open prospective study aimed to evaluate $\mathrm{CH}$ BoNTA treatment efficacy regarding clinical and psychopathological variables in a multidisciplinary setting.

\section{Materials and methods}

We treated $32 \mathrm{CH}$ patients (8 males; 24 females; 44.76 \pm 11.23 mean age) with 190-units BoNTA injections. Sessions took place from January 2014 to June 2015 once every 3 months; patients received headache education; at the baseline (T0) and at the final follow-up (T1) patients completed a headache diary, pain Numerical Rating Scale (NRS), Migraine Disability Assessment (MIDAS), Zung Self-Rating Anxiety Scale (SAS) and Zung Self-Rating Depression Scale (SDS). We considered patients responders if they had $>50 \%$ reduction in headache frequency and/or pain intensity compared with baseline. Using SOFA Statistics 1.4.4 software, we calculated descriptive indicators and evaluated treatment effect using paired-samples Student's $t$-test on clinical and psychosocial variables between $\mathrm{T} 0$ and $\mathrm{T} 1$. We set $\mathrm{p}<0.05$ as threshold of statistical significance.

\section{Results}

At T0 headache frequency (M $\pm \mathrm{DS})$ was $23.71 \pm 5.38$ headache days/month; NRS score was 9.10 \pm 0.94 ; MIDAS score was $57.59 \pm 24.72$; SAS score was $48.38 \pm 12.16$; SDS score was $46.68 \pm 14.31$. At $\mathrm{T} 1$ headache frequency $(\mathrm{M} \pm \mathrm{DS})$ was

\footnotetext{
* Correspondence: centrocefaleeaopc@gmail.com

${ }^{2}$ Centre for Headache and Adaptive Disorders, Unit of Neurology, Department of Neuroscience and Sense Organs, Azienda Ospedaliera

"Pugliese-Ciaccio", Catanzaro, Italy

Full list of author information is available at the end of the article
}

$9.14 \pm 8.31$ headache days/month; NRS score was 6.33 \pm 2.33 ; MIDAS score was $21.517 \pm 20.19$; SAS score was $39.38 \pm 9.19$; SDS score was $36.01 \pm 10.32$. Twenty-one patients $(65.25 \%)$ showed psychopathological comorbidity. We observed response to treatment in 23 patients $(71.87 \%)$ with a statistically significant treatment effect on headache frequency $(t=8.539 ; \mathrm{p}<0.001)$, pain intensity $(t=5.451 ; \mathrm{p}<0.001)$, disability $(t=5.701 ; \mathrm{p}<$ $0.001)$, anxiety $(t=3.457 ; \mathrm{p}<0.005)$ and depression $(t=$ $2.59 ; \mathrm{p}<0.05)$.

\section{Conclusions}

Our data support that BoNTA in a multidisciplinary setting is an effective treatment for chronic headache, potentially addressing pain-related affective disorders. Further developments of our study could evaluate the effectiveness of enhanced psychological interventions, such as stress management, as a complement to the biomedical treatment.

Written informed consent to publish was obtained from the patient(s).

\section{Authors' details}

'Department of Health Science, Magna Graecia University, Catanzaro, Italy.

${ }^{2}$ Centre for Headache and Adaptive Disorders, Unit of Neurology, Department of Neuroscience and Sense Organs, Azienda Ospedaliera

"Pugliese-Ciaccio", Catanzaro, Italy.

Published: 28 September 2015

Reference

1. Cairns BE, Gazerani P: Botulinum neurotoxin A for chronic migraine headaches: does it work and how? Pain Manag 2014, 4(6):377-380.

doi:10.1186/1129-2377-16-S1-A185

Cite this article as: Vescio et al.: P005. Efficacy Type-A Botulinum toxin treatment in a multidisciplinary setting for chronic headache. The Journal of Headache and Pain 2015 16(Suppl 1):A185. creativecommons.org/licenses/by/4.0), which permits unrestricted use, distribution, and reproduction in any medium, provided the original work is properly cited. The Creative Commons Public Domain Dedication waiver (http://creativecommons.org/publicdomain/ zero/1.0/) applies to the data made available in this article, unless otherwise stated. 